\title{
A cost analysis of operative repair of major laparoscopic bile duct injuries
}

\author{
S Hofmeyr, ${ }^{1,2}$ MB ChB, FCS (SA), MMed (Chir), Cert Gastroenterol (SA) Surg; J E J Krige, ${ }^{1,2}$ MB ChB, MSc, FACS, FRCS, FCS (SA); \\ P C Bornman, ${ }^{1,2}$ MB ChB, MMed (Surg), FCS (SA), FRCS (Edin), FRCS (Glasg); S J Beningfield, ${ }^{3}$ MB ChB, FFRad (SA) \\ ${ }^{1}$ Department of Surgery, Faculty of Health Sciences, University of Cape Town, South Africa \\ ${ }^{2}$ Surgical Gastroenterology Unit, Groote Schuur Hospital, and University of Cape Town Private Academic Hospital, Cape Town, South Africa \\ ${ }^{3}$ Department of Radiology, Faculty of Health Sciences, University of Cape Town, South Africa
}

Corresponding author: S Hofmeyr (stefhofmeyr@sun.ac.za)

Background. Major bile duct injuries occur infrequently after laparoscopic cholecystectomy, but may result in life-threatening complications. Few data exist on the financial implications of duct repair. This study calculated the costs of operative repair in a cohort of patients who underwent reconstruction of the bile duct after major ductal injury.

Objective. To calculate the total in-hospital cost of surgical repair of patients referred with major bile duct injuries.

Methods. A prospective database was reviewed to identify all patients referred to the University of Cape Town Private Academic Hospital, South Africa, between 2002 and 2013 for assessment and repair of major laparoscopic bile duct injuries. The detailed clinical records and billing information were evaluated to determine all costs from admission to discharge. Total costs for each patient were adjusted for inflation between the year of repair and 2013. Results. Forty-four patients (33 women, 11 men; median age 48 years, range 30 - 78) underwent reconstruction of a major bile duct injury. First-time repairs were performed at a median of 24.5 days (range 1 - 3 662) after initial surgery. Median hospital stay was 15 days (range 6 86). Mean cost of repair was ZAR215 711 (range ZAR68 764 - 980 830). Major contributors to cost were theatre expenses (22\%), admission to intensive care (21\%), radiology (17\%) and specialist fees (12\%). Admission to a general ward (10\%), consumables (7\%), pharmacy (5\%), endoscopy (3\%) and laboratory costs $(3 \%)$ made up the balance.

Conclusions. The cost of repair of a major laparoscopic bile duct injury is substantial owing to prolonged hospitalisation, complex surgical intervention and intensive imaging requirements.

S Afr Med J 2015;105(6):454-457. DOI:10.7196/SAMJ.9038

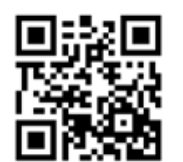

Although laparoscopic cholecystectomy was introduced more than two decades ago, the incidence of bile duct injuries has not diminished and injury still occurs in $0.4 \%$ of operations, twice as often as with open cholecystectomy. ${ }^{[1-4]}$ While minor injuries with duct continuity can be treated successfully with endoscopic stenting without recourse to operation, major injuries with duct division are life-threatening and may require complex biliary reconstructive surgery. ${ }^{[5]}$ Optimal evaluation of a major bile duct injury requires careful, co-ordinated, multidisciplinary assessment by a knowledgeable group of surgeons, intensivists, endoscopists and interventional radiologists. ${ }^{[6,7]}$ Reparative biliary surgery is technically demanding and should be undertaken only by a surgical team with expertise and established credentials.

The implications of a major bile duct injury can be profound, with the spectre of protracted hospitalisation and invasive investigations, the anxiety of major reconstructive surgery, a lengthy rehabilitation period, decreased quality of life, loss of income, and in some cases prolonged and unpleasant litigation. The financial burden implicit in injury management and the consequences for the patient are significant, yet no local and few international data are available to assess the cost of definitive bile duct reconstruction accurately. ${ }^{[8-10]} \mathrm{In}$ this study we calculated the total in-hospital costs of definitive repair of major laparoscopic bile duct injuries by including all costs incurred from referral to discharge from hospital with a durable repair.

\section{Methods}

\section{Study population}

Between March 2002 and October 2013, 52 patients were referred to the University of Cape Town Private Academic Hospital (UCTPAH),
South Africa (SA), for assessment of a suspected or confirmed major injury to the extrahepatic bile duct sustained during a laparoscopic cholecystectomy. Information was obtained from a prospective database on all patients with a laparoscopic bile duct injury that is maintained in the Surgical Gastroenterology Unit, Groote Schuur Hospital, Cape Town, by a dedicated research assistant. Data collected and entered included patient demographics, indication for laparoscopic cholecystectomy, recognition of injury during or subsequent to cholecystectomy, mode of delayed presentation, delay in referral, investigations and procedures performed before referral, type of injury according to the Strasberg classification, ${ }^{[2]}$ investigations and procedures prior to definitive surgery, timing of repair, length of intensive care unit (ICU) and total hospital stay, investigations and procedures after repair, and complications. A cohort of 44 patients who had operative repair of a major bile duct injury was identified and analysed. Eight patients were excluded because they had a minor bile duct injury that did not require surgery $(n=2)$ or complete and detailed billing information was not available on the hospital computer records $(n=6)$. The information captured on the database, as well as the detailed original clinical notes, was reviewed. The study was approved by the University of Cape Town Health Research Ethics Committee (HREC REF 600/2014).

Each patient underwent detailed preoperative assessment to define the extent of the bile duct injury, including a multiphase computed tomography (CT) scan, magnetic resonance cholangiopancreatography (MRCP) and percutaneous transhepatic cholangiography (PTC) with biliary drain placement. Possible arterial injuries were identified by a contrast-enhanced CT scan and duplex Doppler flow assessment if required. 
The interval from initial injury to definitive repair was defined as the number of days from initial injury to definitive repair at UCTPAH. This interval was inclusive of previous repairs performed at outside medical centres. For the purpose of the study, 'first repairs' were defined as patients without a previous attempt at repair or an attempted repair by any method other than a bilioenteric anastomosis. 'Revision surgery' was the term used when a repair was performed on patients with a previous hepatico- or choledochojejunostomy.

Biliary injuries were classified using the Strasberg classification of bile duct injuries: ${ }^{[2]}$ type A - bile leak from cystic duct stump or gallbladder bed; type B - aberrant right hepatic duct occlusion; type $\mathrm{C}-$ aberrant right hepatic duct transection; type D partial $(<50 \%)$ transection of a major bile duct; and type $\mathrm{E}->50 \%$ transection or complete transection of a major bile duct (further subclassified as E1 $->2 \mathrm{~cm}$ from the confluence of the left and right hepatic ducts; E2 - <2 cm from confluence; E3 - no common hepatic duct remnant with an intact hepatic duct confluence; E4 - destruction of the hepatic duct confluence; and E5 aberrant right sectoral duct in conjunction with an injury to the common hepatic duct).

\section{Operative technique}

A standard operative technique was used for all bile duct reconstructions. The full technical details have been published previously. ${ }^{[11]}$ In brief, a bilateral subcostal incision $3 \mathrm{~cm}$ below the costal margin was used. An Omnitract fixed body wall retractor provided exposure to the upper abdomen. All adhesions in the right upper quadrant were dissected free and released. The hepatic arterial and portal venous vasculature in the hepatoduodenal ligament was identified and preserved. The site of the bile duct injury was identified. In patients with a complete duct transection, the previously placed percutaneous transhepatic biliary drains were located. All fibrotic tissue in the proximal hepatoduodenal ligament adjacent to the injury was excised. The hepatic ducts identified at the level of the hepatic duct confluence were exposed by incising the hilar plate at the base of the quadrate lobe and lowering the extrahepatic left hepatic duct and the hepatic duct confluence. The ducts were dissected until healthy, well-vascularised ductal mucosa was identified. An anterior longitudinal incision was made in the extrahepatic component of the left hepatic duct using the Hepp-Couinaud approach. Careful choledochoscopy was done to identify the right and left segmental ducts and ensure the absence of intrahepatic stones. The operative choledochoscopic findings were reconciled with the preoperative MRCP and PTC imaging to ensure identification of all ducts. A $40 \mathrm{~cm}$ retrocolic jejunal Rouxen-Y loop was fashioned and a side-to-side hepatojejunal anastomosis constructed using preplaced 5/0 absorbable monofilament sutures. The hepatojejunal anastomoses were stented using the existing percutaneous transhepatic biliary catheters. A side-to-side enteroanastomosis was done in the infracolic compartment. One week postoperatively, percutaneous cholangiography was performed via the biliary drains to confirm an intact biliary-enteric anastomosis. The percutaneous drains were removed 14 days later. Postoperative complications were graded according to the Clavien-Dindo classification system. ${ }^{[12]}$

\section{Calculation of financial data}

All patient costs from admission to discharge, as captured daily into the hospital billing system, were accessed. The complete hospital charge sheet, as well as the invoices for laboratory investigations, radiology services and all specialist clinicians contributing to care, were reviewed and aggregated to calculate the total cost per patient. Costs were classified under the following categories: hospital bed costs, which were subdivided into general ward, ICU and high care; cost of ward consumables (swabs, dressings, intravenous cannulas and lines, etc); pharmacy costs; operating theatre costs (theatre time, consumables including sutures, anaesthetic gases, etc.); radiology costs; laboratory costs and specialist fees. The high inflation rate of healthcare expenditure in SA during the study period from 2002 to 2013 exceeded consumer price inflation, and necessitated adjustment of the calculated costs for each year to 2013 figures before a meaningful statistical analysis could be performed. Costs were adjusted with data specific to medical inflation available from Statistics South Africa (www.statssa.gov.za).

\section{Statistical analysis}

Data were analysed with Microsoft Excel and results presented as percentages, medians, means and ranges. STATA version 11 was used to study the correlation between postoperative complications, sepsis on admission, conversion to open surgery upon recognition and cost of repair, by applying the $\chi^{2}$ test.

\section{Results}

During the study period, 44 patients (33 women, 11 men; median age 48 years, range 30 - 78) with major bile duct injuries were assessed and the injuries repaired (Table 1). The indication for laparoscopic cholecystectomy was symptomatic gallstones in $80 \%$ of patients and acute cholecystitis in $20 \% ; 43 \%$ of injuries were recognised during the index operation. Patients were referred for evaluation and management of new injuries after a median of 14.5 days (range 1 3662 ), and definitive repair was performed at a median of 24.5 days after injury (range 1 - 3 674). Nine repairs (26\%) were done within 7 days of the injury, 6 (18\%) between 7 and 14 days, $6(18 \%)$ between 2 and 6 weeks and $13(38 \%)$ after 6 weeks. Strictures of 10 previous repairs done elsewhere required reoperation at a median of 5 years (range 240 days - 16 years) after the initial repair. Patients spent a median of 15 days (range 6 - 86) in hospital, of which a median of 5.5 days $(2-55)$ were in the high-care unit or ICU. There were no perioperative deaths. Two patients $(5 \%)$ had Strasberg type C injuries, 6 (14\%) type E1 injuries, 33 (75\%) type E2 injuries, 2 (5\%) type E3 injuries and $1(2 \%)$ a type E4 injury. Theatre and ICU/high-care admission were the major

\section{Table 1. Patient demographics $(N=44)$ and general data}

\begin{tabular}{ll}
\hline Age (years), median (range) & $48(30-78)$ \\
Males, $n(\%)$ & $11(25.0)$ \\
Females, $n(\%)$ & $33(75.0)$ \\
Indication for cholecystectomy: uncomplicated symptomatic gallstones, $n(\%)$ & $35(79.5)$ \\
Indication for cholecystectomy: acute cholecystitis, $n(\%)$ & $9(20.4)$ \\
Injury recognised at cholecystectomy, $n(\%)$ & $19(43.2)$ \\
Injury not recognised at cholecystectomy, $n(\%)$ & $25(56.8)$ \\
Total days in hospital, median (range) & $15(6-86)$ \\
Days in ICU/high care, median (range) & $5.5(2-55)$ \\
Days in general ward, median (range) & $9(2-46)$
\end{tabular}


contributors to cost, accounting for $22 \%$ and $21 \%$ of the total costs of repair, respectively. The contributors to cost are summarised in Table 2. The inflation-adjusted mean total cost of repair was ZAR215 711 (range ZAR68 764 - 980 830).

The data were scrutinised to detect factors that were responsible for increasing the cost of repairing new bile duct injuries. The correlations between the cost of repair and the presence of sepsis on admission (cholangitis, infected bilomas, biliary peritonitis), postoperative complications and conversion to open surgery after recognition at the index laparoscopic cholecystectomy are illustrated in Table 3. The association between these factors and increased cost was not statistically significant, probably owing to type 2 statistical error. Fifteen postoperative complications occurred in 14 patients (32\%), as shown in Table 4. Patients with postoperative complications spent a median of 23 total days in hospital, as opposed to a median of 13 total days in hospital for those without complications. On multivariate analysis, delay in referral did not appear to influence the cost of repair.

\section{Discussion}

The benefits of laparoscopic over traditional open cholecystectomy include less postoperative pain, shorter hospital stay and smaller incisions, thus avoiding the sequelae of large abdominal
Table 2. Contributors to total cost of definitive repair

\begin{tabular}{lll}
\hline & $\%$ & Mean cost (ZAR) \\
\hline Theatre & 22.2 & 47883 \\
Bed (ICU/high care) & 20.9 & 44985 \\
Radiology & 17.3 & 37259 \\
Specialist fees & 12.3 & 26595 \\
Bed (general ward) & 9.9 & 21322 \\
Ward consumables & 6.5 & 14053 \\
Pharmacy & 5.0 & 10785 \\
Endoscopy & 2.9 & 6359 \\
Laboratory & 2.9 & 6469 \\
Total & & 215711
\end{tabular}

Table 3. Factors associated with increased cost of repair

\begin{tabular}{|c|c|c|c|}
\hline & $n$ & Cost (ZAR) & $p$-value \\
\hline Mean cost of new repairs & 34 & 230452 & \multirow{2}{*}{$0.44^{*}$} \\
\hline Mean cost of revision surgery & 10 & 165589 & \\
\hline Mean cost of new repair (recognised, not converted) & 7 & 175349 & \multirow{2}{*}{$0.42^{*}$} \\
\hline Mean cost of new repair (recognised, converted) & 6 & 350611 & \\
\hline Mean cost without postoperative complications & 35 & 208606 & \multirow[b]{2}{*}{$0.43^{*}$} \\
\hline $\begin{array}{l}\text { Mean cost with postoperative complications (> grade } 1 \text { Clavien- } \\
\text { Dindo }^{[12]} \text { ) }\end{array}$ & 9 & 243338 & \\
\hline Mean cost if presented with sepsis & 10 & 317949 & \multirow{2}{*}{$0.35^{\star}$} \\
\hline Mean cost if presented without sepsis & 34 & 185641 & \\
\hline
\end{tabular}

Table 4. Summary of postoperative complications

\begin{tabular}{lll}
\hline Grade according to Clavien-Dindo system ${ }^{[12]}$ & $\boldsymbol{n}$ & Description \\
\hline Grade 1 & 5 & Wound sepsis \\
Grade 2 & 2 & Wound sepsis \\
Grade $3 \mathrm{a}$ & 2 & Perihepatic abscess, perihepatic abscess \\
Grade $3 \mathrm{~b}$ & 3 & Biloma, incisional hernia, early \\
& & postoperative small-bowel obstruction \\
Grade 4 & 2 & Myocardial infarction, cerebrovascular accident
\end{tabular}

wall incisions. ${ }^{[1]}$ The major disadvantage of the laparoscopic technique is the increased incidence of bile duct injuries, which may result in considerable morbidity. ${ }^{[1-4]}$ Healthrelated quality of life after bile duct injury in comparison with uncomplicated laparoscopic cholecystectomy has been shown to be adversely affected as measured by psychological outcome, while physical outcomes appear to be similar. ${ }^{[13]}$

Little information is available to accurately quantify the overall financial implications of a bile duct reconstruction, incorporating such diverse costs as loss of income due to time off work, travel expenses, medical fees, rehabilitation and possible litigation. Loss of income encompasses not only time away from work while in hospital, but also the convalescent period and follow-up visits and may extend to a partner or spouse involved in a supportive capacity who may need to travel long distances between home and the hospital where the repair is done. These costs are, by their very nature, difficult to calculate accurately and little information on medicolegal costs is available, ${ }^{[14]}$ especially locally.

The cost of definitive bile duct reconstruction has been reported on previously, ${ }^{[8-10]}$ with Savader et al. ${ }^{[8]}$ reporting a mean cost of USD51 411 in 1995, ranging from 4.5 to 26.0 times the cost of an uncomplicated laparoscopic cholecystectomy. The cohort of 44 patients in our study who all had major bile duct injuries repaired by Roux-en-Y hepaticojejunostomy is unique with respect to the number of patients evaluated and the inclusion of all applicable costs plus adjustment for inflation, as well as inclusion of the time from hospital admission for the definitive repair to discharge. The mean cost of ZAR215 711 is substantial and is 6.4 times the cost of an uncomplicated laparoscopic cholecystectomy performed at the same institution. The most expensive repair in this study amounted to ZAR980 830, incorporating 86 days spent in hospital, which illustrates the potential impact of bile duct injuries and the high cost imposed on medical insurance and/or patients.

Early recognition of bile duct injury and referral to a hepatobiliary surgeon are essential to reduce morbidity and ensure a satisfactory surgical outcome. ${ }^{[15]}$ Yet in this study, $57 \%$ of injuries were only recognised after a median delay of 5 days, with 19 of 25 patients presenting with sepsis due to cholangitis or biliary peritonitis or septic bilomas, a finding similar to previously reported data. ${ }^{[7]}$ These findings reinforce the maxim that all laparoscopic cholecystectomy patients require careful assessment and thorough investigation to exclude iatrogenic injury to the bile ducts in the event of any unexpected postoperative symptoms. 
For methodological reasons it was not possible to calculate costs incurred at the hospital where the injury occurred. Accumulated costs before referral were estimated to be substantial in some cases, including specialised imaging, attempted repair of the injury, or reoperation for intraperitoneal sepsis.

Correlations with the cost of repair were studied with the aim of identifying those modifiable factors responsible for driving up the cost of repair. Intuitively, delayed recognition, sepsis and complications ought to have an adverse effect on the eventual outcome and cost of repairing a bile duct injury owing to increased length of ICU and hospital stay, increased imaging investigations and interventions to address intra-abdominal sepsis. However, these factors could not be proven to have a statistically significant effect on cost in this study.

In conclusion, bile duct injuries after laparoscopic cholecystectomy remain a serious problem in modern surgical practice. There is general consensus that patients who require evaluation and repair of a bile duct injury should be referred to a specialised centre. The present study shows that in a tertiary academic centre, reconstructive surgery for complex iatrogenic laparoscopic bile duct injuries has acceptable morbidity and can be accomplished with no mortality. The costs incurred as a consequence of a bile duct injury are considerable and result in a substantial economic burden. The absolute aggregated cost of a bile duct repair is dependent on a variety of factors. This study reflects the experience of a high-volume referral centre, and extrapolation to other centres may not be applicable. As the consequences of a bile duct injury can be devastating, prevention must remain the top priority during laparoscopic cholecystectomy.

\section{References}

1. Smith JF, Boysen D, Tschirhart J, et al. Comparison of laparoscopic cholecystectomy versus elective ope cholecystectomy. J Laparoendosc Surg 1992;2(6):311-317. [http://dx.doi.org/10.1089/lps.1992.2.311]

2. Strasberg SM, Hertl M, Soper NJ. An analysis of the problem of biliary injury during laparoscopic cholecystectomy. J Am Coll Surg 1995;189:101-125.

3. Richardson MC, Bell G, Fullarton GM. Incidence and nature of bile duct injuries followin laparoscopic cholecystectomy: An audit of 5913 cases. Br J Surg 1996;83(10):1356-136. [http://dx.doi. org/10.1002/bjs.1800831009]

4. Nuzzo G, Giulante F, Giovannini I, et al. Bile duct injury during laparoscopic cholecystectomy: Results of an Italian national survey on 56591 cholecystectomies. Arch Surg 2005;140(10):986-992. [http:// dx.doi.org/10.1001/archsurg.140.10.986

5. De Reuver RP, Rauws EA, Vermeulen M, et al. Endoscopic treatment of post-surgical bile duct injuries: Long term outcome and predictors of success. Gut 2007;56(11):1599-1605. [http://dx.doi.org/10.1136/ Long term outcome and predictors of success. Gut 2007;56(11):1599-1605. [http://dx.doi.org/10.1136/ gut.2007.123596

6. Connor S, Garden OJ. Bile duct injury in the era of laparoscopic cholecystectomy. Br J Surg 2006;93(2):158-168. [http://dx.doi.org/10.1002/bjs.5266]

7. Sicklick JK, Camp MS, Lillemoe KD, et al. Surgical management of bile duct injuries sustained durin laparoscopic cholecystectomy: Perioperative results in 200 patients. Ann Surg 2005;241(5):786-792. [http://dx.doi.org/10.1097/01.sla.0000161029.27410.71]

8. Savader SJ, Lillemoe KD, Prescott CA, et al. Laparoscopic cholecystectomy-related bile duct injuries A health and financial disaster. Ann Surg 1997:225(3):268-273. [http://dx.doi.org/10.1097/00000658199703000-00005]

9. Andersson K, Eriksson K, Blind P. Iatrogenic bile duct injury - a cost analysis. HPB 2008;10(6):416419. [http://dx.doi.org/10.1080/13651820802140745]

10. Woods MS. Estimated costs of biliary tract complications in laparoscopic cholecystectomy based upon Medicare cost/charge ratios: A case-control study. Surg Endosc 1996;10(10):1004-1007. [http://dx.doi. Medicare cost/charge ratios: $A$ ca

11. Terblanche J, Worthley CS, Spence RA, Krige JE. High or low hepaticojejunostomy for bile duct strictures. Surgery 1990;108(5):828-834.

12. Dindo D, Demartines N, Clavien PA. Classification of surgical complications: A new proposal with evaluation in a cohort of 6336 patients and results of a survey. Ann Surg 2004:240(2):205-213. [http:// dx.doi.org/10.1097/01.sla.0000133083.54934.ae]

13. Landman MP, Feurer ID, Moore DE, et al. The long-term effect of bile duct injuries on health-related quality of life: A meta-analysis. HPB 2013;15(4):252-259. [http://dx.doi.org/10.1111/j.1477-2574.2012.00586.x]

14. Roy PG, Soonawalla ZF, Grant HW. Medicolegal costs of bile duct injuries incurred during laparoscopic cholecystectomy. HPB 2009;11(2):130-134. [http://dx.doi.org/10.1111/j.1477-2574.2008.00023.x)

15. Thomson BN, Parks RW, Madhavan KK, et al. Early specialist repair of biliary injury. Br J Surg 2006;93(2):216-220. [http://dx.doi.org/10.1002/bjs.5194]

Accepted 23 October 2014. 